\title{
ASYMPTOTIC BEHAVIOR OF RADIAL MINIMIZERS OF A $p$-ENERGY FUNCTIONAL WITH NONVANISHING DIRICHLET BOUNDARY CONDITION
}

\author{
YAQIN ZHENG AND YUTIAN LEI
}

Abstract. This paper is concerned with a $p$-energy functional with nonvanishing Dirichlet boundary condition. The authors prove the $W_{l o c}^{1, p}$ convergence of the radial minimizer, and discuss the location of the zeros of this minimizer. In addition, an estimate of the convergence rate of the minimizer is given by means of the iterative approach.

Mathematics subject classification (2000): 35J70, 49K20.

Keywords and phrases: $p$-energy functional, radial minimizer, $W_{l o c}^{1, p}$ convergence, location of the zeros, estimate of the convergence rate.

\section{REFERENCES}

[1] F.Bethuel, H.Brezis, F.Helein, Ginzburg-Landau Vortices, Birkhäuser, Berlin, 1994.

[2] Z.C.HAN, Y.Y.LI, Degenerate elliptic systems and applications to Ginzburg - Landau type equations, Part I, Calc. Var. Partial Differential Equations, 4 (1996), 171-202.

[3] F.B.HAng, F.H.Lin, Static theory for planar ferromagnets and antiferromagnets, Acta. Math. Sin. (Engl.Ser.), 17 (2001), 541-580.

[4] R.HardT, F.H.Lin, Mappings minimizing the $L^{p}$ norm of the gradient, Comm. Pur. Appl. Math., 40 (1987), 555-588.

[5] M.C.Hong, Asymptotic behavior for minimizers of a Ginzburg - Landau type functional in higher dimensions associated with $n$-harmonic maps, Adv. Differential Equations, 1 (1996), 611-634.

[6] S.Komineas, N.PAPANicolaou, Vortex dynamics in two-dimensional antiferromagnets, Nonlinearity, 11 (1998), 265-290.

[7] Y.T.LEI, Asymptotic analysis of the radial minimizers of an energy functional, Methods Appl. Anal., 10 (2003), 67-80.

[8] Y.T.LEI, Asymptotic behavior of the regularized minimizer of an energy functional in higher dimensions, J. Math. Anal. Appl., 334 (2007), 1341-1362.

[9] Y.T.LEI, Radial minimizers of p-Ginzburg-Landau type with $p \in(n-1, n)$, Nonlinear Anal., 69 (2008), 4534-4549.

[10] N.PAPAnicolaou, P.N.Spathis, Semitopological solutions in planar ferromagnets, Nonlinearity, 12 (1999), 285-302. 